\title{
Socio-demographic characteristics of patients referred to a National Health Service Adult Weight Management Service: a retrospective observational study
}

\author{
E.M. Di Battista ${ }^{1}$, D.A. Behrens ${ }^{2}$ and T. Filipponi ${ }^{3}$ \\ ${ }^{1}$ Adult Weight Management Service, Aneurin Bevan University Health Board, Newport, Wales, \\ ${ }^{2}$ Aneurin Bevan Continuous Improvement Department, Aneurin Bevan University Health Board, Newport, Wales and \\ ${ }^{3}$ Faculty of Life Sciences and Education, University of South Wales, Treforest, Wales
}

In the last thirty years, the number of people with obesity has more than doubled worldwide (WHO) ${ }^{(1)}$. The latest figures from the Welsh Health Survey (2015) suggests average obesity rates of $24 \%$ with increased obesity rates of up to $29 \%$ in urban areas of lowersocioeconomic status $\left(\right.$ LSES) ${ }^{(2)}$. In response, Aneurin Bevan University Health Board (ABUHB) implemented an Adult Weight Management Service (AWMS) in January 2014. In order to inform service provision and support the development of an equitable obesity strategy for ABUHB, the aim of this study was to understand the socio-demographic characteristics of those adult citizens referred to AWMS.

Permission to conduct the study was granted by ABUHB's Research Risk Review Committee. Patients who were referred into ABUHB's AWMS and attended the first appointment $(n=2606)$ between January 2014 and December 2016 were included in the analysis. The majority of these patients $(n=2,557)$ resided in Gwent and South Powys. Data cleansing was carried out and 106 invalid records removed. Units of analysis for the study were (i) the Lower Super Output Area (LSOA) derived from the postcode of AWMS patient residence, and (ii) patient Body Mass Index (BMI). For the 2,500 valid patient records LSOAs were related to Welsh Index of Multiple Deprivation (WIMD) ranks [5] (with rank 1 being assigned to the most deprived LSOA, and rank 1909 to the least deprived LSOA). To test for correlation with patient BMI, both the WIMD ranks from 1-1909 and an aggregation into quintiles were used (with the first quintile corresponding to the most deprived regions, quintile 5 to the least deprived ones); performing a One-Way ANOVA for the latter.

The majority of patients $(n=2312 ; 92.5 \%)$ identified themselves as White British. Total number of males and females attending the AWMS and included in the analyses were $726(29 \%)$ and $1774(71 \%)$, respectively. Overall, patient age was normally distributed with a mean of 50.2 years - gender analysis suggests average male age of AWMS patients was however significantly older than females $[53.7$ years $(\mathrm{SD}=14.2)$ and 48.8 years $(\mathrm{SD}=14.9)$, respectively; $\mathrm{p}<0.001]$. Average BMI for both males and females was $43.5 \mathrm{~kg} / \mathrm{m}^{2}$ (SD = 8.7); the most frequently observed BMI was however gender sensitive at $33.1 \mathrm{~kg} / \mathrm{m}^{2}$ for female and $41.6 \mathrm{~kg} / \mathrm{m}^{2}$ for male patients. The relationship between patient BMI and patient WIMD rank illustrates that these two quantities were not correlated among AWMS patients $(r=0.03$; significant at the $10 \%$ level).

Findings suggest that patients referred into a weight management service implemented in urban areas may not have a lowsocioeconomic background according to the WIMD rank. The AWMS may therefore not favour LSES groups to access the service, so in order to address these inequalities, the AWMS could benefit from service reconfiguration of location planning and referral pathways. Additionally, the results indicate that males and patients from ethnic minorities are currently less likely to attend the AWMS. These findings could warrant the development of gender/ethnicity specific, equity-focused, weight management interventions.

1. World Health Organisation (2014). Global status report on noncommunicable diseases 2014. Geneva: WHO

2. Public Health Wales Observatory (2015) - Obesity: Overview. http://www.publichealthwalesobservatory.wales.nhs.uk/obesity-overview (accessed April, 2017)

3. Welsh Government (2015) - Welsh Index of Multiple Deprivation. http://gov.wales/statistics-and-research/welsh-index-multiple-deprivation/?lang =en (accessed April, 2017) 